\title{
Le personnage du fanfaron: théâtre récits cinéma, dir. Él. GAVOILLE et C. TERRILE
}

\section{Roberta Sapino}

\section{(2) OpenEdition}

\section{Journals}

\section{Edizione digitale}

URL: https://journals.openedition.org/studifrancesi/45650

DOI: 10.4000/studifrancesi.45650

ISSN: 2421-5856

\section{Editore}

Rosenberg \& Sellier

\section{Edizione cartacea}

Data di pubblicazione: 1 août 2021

Paginazione: 423-424

ISSN: 0039-2944

\section{Notizia bibliografica digitale}

Roberta Sapino, «Le personnage du fanfaron: théâtre récits cinéma, dir. Él. GavollLe et C. TERRILE», Studi Francesi [Online], 194 (LXV | II) | 2021, online dal 01 septembre 2021, consultato il 15 octobre 2022. URL: http://journals.openedition.org/studifrancesi/45650 ; DOI: https://doi.org/10.4000/studifrancesi. 45650

Questo documento è stato generato automaticamente il 15 octobre 2022

\section{(c) (i) (9)}

Creative Commons - Attribuzione - Non commerciale - Non opere derivate 4.0 Internazionale - CC BY NC-ND 4.0

https://creativecommons.org/licenses/by-nc-nd/4.0/ 


\title{
Le personnage du fanfaron: théâtre récits cinéma, dir. Él. GAVOILLE et $\mathrm{C}$. TERRILE
}

\author{
Roberta Sapino
}

\section{NOTIZIA}

Le personnage du fanfaron: théâtre récits cinéma, dir. Él. GAVOILLE et C. TERRILE, Paris, Éditions Kimé, 2020, 274 pp.

1 Il fanfarone ha attraversato i secoli: dall'alazôn greco e dal gloriosus latino fino alle declinazioni contemporanee, la sua figura caratterizzata dall'eccesso, dalla vanità, dall'ostentazione e capace di toccare, oltre al registro del burlesco, anche toni tragici, ha assunto tratti e valori diversi a seconda dei contesti socioculturali e delle sensibilità autoriali di cui è emanazione. Proprio di questa persistenza e malleabilità rende conto il ricco volume curato da Élisabeth GAVOILLE e Cristina TERRILE, sviluppo in forma editoriale di un dialogo tra studiosi cominciato in un convegno dedicato al fanfaron organizzato nel 2018 all'Università di Tours (Élisabeth Gavoille e Cristina Terrile, Remerciements e Introduction, p. 7 e pp. 9-17).

2 Se le prime due sezioni avviano un percorso cronologico che si conclude, nella quarta, con il Novecento, la terza affronta casi in cui è l'istanza narrante a presentare i tratti caratteristici del fanfaron e introduce questioni teoriche utili per meglio comprendere le ultime pagine.

3 Nella sezione iniziale, intitolata «Le modèle antique du soldat fanfaron», Nathalie LHoSTis compara due testi di Aristofane e di Plauto per dimostrare quanto l'attualità politica incida sulla caratterizzazione del personaggio (Faire du fanfaron un spectacle: le Lamachos d'Aristophane et le Pygropolinice de Plaute, pp. 21-38); Élisabeth GAVOILLE studia i meccanismi che sottendono le coppie di soldati fanfaroni e parassiti costituite da Pigropolinice e Artotrogo e da Trasone e Gnatone (Le soldat fanfaron et son parasite chez 
Plaute et Térence, pp. 39-58); Émeline MARQUIS mette in luce come il soldato fanfarone sia sottoposto, nei Dialoghi delle cortigiane di Luciano, a un triplo sguardo: delle cortigiane, dell'autore, del lettore - e il primo non è per lui, conclude la studiosa, il più pericoloso (À l'aune du regard féminin? Le soldat fanfaron dans les "Dialogues des courtisanes" de Lucien, pp. 59-73).

Il capitolo «Fanfarons renaissants et baroques» si apre con un contributo in cui Patrizia FARINELLI, muovendo da considerazioni di Massimo Bontempelli e di Mario Baratto, fa notare come la Talanta si differenzi dalla produzione precedente di Pietro Aretino in virtù dello sfoggio di un'abilità retorica priva di valore sovversivo (Facture maniériste $d u$ 'miles' chez l'Arétin: le capitaine Tinca de la "Talanta", pp. 77-94). Alice VINTENON osserva che la missione pedagogica riconosciuta alla commedia nel Cinquecento ha contribuito a limitare l"'imagination verbale" attribuita al fanfarone (La représentation du fanfaron à la Renaissance, entre 'imitatio vitae' et fantaisie romanesque, pp. 95-111), mentre Françoise POULET mostra che tra la fine del Cinquecento e l'inizio del secolo successivo l'attribuzione di un'origine e quindi di una lingua straniera al personaggio del soldato fanfarone è una strategia di delegittimazione che si inscrive in una più ampia riflessione sul principio dell'honnêteté (Les rodomontades du fanfaron: imaginaire d'une langue tyrannique (fin XVI $\mathrm{e}^{\mathrm{e}}$ début du XVII $\mathrm{I}^{\mathrm{e}}$ siècle), pp. 113-127). Florent GABAUDE porta l'attenzione sulle metafore avicole usate per descrivere il "fanfaron protomoderne" in area germanica: ali, piume e cinguettii evocano modalità di spostamento, particolarità dell'aspetto e forme espressive ben connotate (Volubiles volatiles: l'image des fanfarons dans la littérature allemande de la première modernité, pp. 129-151). In De la bibliothèque au personnage: Donna Quinzia, la fanfaronne, et Baltramina, l'anti-fanfaronne, dans les comédies en lombard de Carlo Maria Maggi (1630-1699), Massimo scandola afferma che l'opera di Maggi propone non solo il nuovo archetipo femminile della fanfaronne, ma anche una rappresentazione "ethnographique" della società milanese di epoca spagnola (pp. 153-168).

5 Élisabeth GAVoILLE firma, oltre all'introduzione e al saggio già menzionato, anche il contributo che introduce la terza sezione, chiamata «Discoureurs et narrateurs fanfarons»: in Un professeur fanfaron: le personnage du 'magister amoris' dans l'“Art d'aimer" d'Ovide, la studiosa ci riporta nell'antichità per indagare le strategie discorsive che fanno del magister amoris ovidiano un dominus che non esita a mostrarsi despotico e vanitoso (pp. 171-185). Con un balzo geografico e temporale giungiamo nell'America dell'Ottocento: il contributo di Stéphanie CARREZ analizza il gioco complesso di voci e di livelli narrativi in Mr. Higginbotham's Catastrophe ponendolo a confronto con la tradizione delle tall tales (Info ou intox? Dominicus Pike, ou le colporteur fanfaron chez Nathaniel Hawthorne, pp. 187-206). A seguire, Gaëlle DEBEAUX si interroga sull' ethos narratoriale (definito sulla base delle teorie di Dominique Maingueneau) individuabile in Jacques le fataliste e in Se una notte d'inverno un viaggiatore: la tendenza del narratore alla fanfaronnade sollecita il lettore ad assumere una postura di ricezione attiva, "à apprendre à lire" (Étude comparée de deux narrateurs fanfarons: "Jacques le fataliste et son maittre" de Denis Diderot et "Si une nuit d'hiver un voyageur" d'Italo Calvino, pp. 207-222).

Chiude il volume il capitolo intitolato «Avatars modernes et interprétations politiques: un personnage symptôme». Proust, Musil, Svevo e Woolf sono tra i principali, seppur non gli unici, riferimenti dello studio di Florence GODEAU, che mostra come anche nel primo Novecento le varie incarnazioni del fanfarone costituiscano delle forme privilegiate per l'analisi e la discussione di rapporti sociali e assunti culturali (Fanfarons 
et anti-héros dans le roman moderniste: «une mystérieuse maladie d'époque»?, pp. 225-236). Marco CARMELlo illustra l'operazione di "riattivazione" del modello antico compiuta da Gadda in Eros e Priapo, testo non antifascista ma "postfascista" secondo le parole dello studioso, dove dietro alla maschera del fanfarone si cela il personaggio storico di Mussolini (Monsieur Pygropolinice ou Mussolini selon Gadda. Une lecture de "Eros e Priapo", pp. 237-249). Si rimane in Italia con l'ultimo contributo, firmato da Luigi SALA e dedicato ad Alberto Sordi: la tendenza dell'attore a intervenire sui copioni e a improvvisare, imponendo il proprio istrionismo e la propria comicità cinica fino a obbligare il regista a riformulare il senso stesso del film, insieme al tipo di personaggio da lui privilegiato, fa di lui un fanfarone dai tratti nichilisti (Alberto Sordi: un fanfaron destructeur, pp. 251-263). 